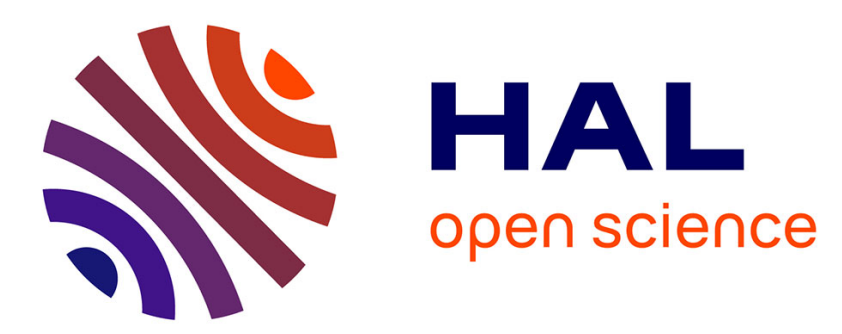

\title{
Wave-Front Correction Methods for Extreme-Ultraviolet Multilayer Reflectors
}

\author{
Mandeep Singh, Matthieu F. Bal, Joseph J. M. Braat, Denis Joyeux, Udo
} Dinger

\section{> To cite this version:}

Mandeep Singh, Matthieu F. Bal, Joseph J. M. Braat, Denis Joyeux, Udo Dinger. Wave-Front Correction Methods for Extreme-Ultraviolet Multilayer Reflectors. Applied optics, 2003, 42 (10), pp.18471851. 10.1364/AO.42.001847 . hal-00875710

\section{HAL Id: hal-00875710 \\ https://hal-iogs.archives-ouvertes.fr/hal-00875710}

Submitted on 22 Oct 2013

HAL is a multi-disciplinary open access archive for the deposit and dissemination of scientific research documents, whether they are published or not. The documents may come from teaching and research institutions in France or abroad, or from public or private research centers.
L'archive ouverte pluridisciplinaire HAL, est destinée au dépôt et à la diffusion de documents scientifiques de niveau recherche, publiés ou non, émanant des établissements d'enseignement et de recherche français ou étrangers, des laboratoires publics ou privés. 


\title{
Wave-front correction methods for extreme-ultraviolet multilayer reflectors
}

\author{
Mandeep Singh, Matthieu F. Bal, Joseph J. M. Braat, Denis Joyeux, and Udo Dinger
}

\begin{abstract}
In this theoretical study we show that by removing or depositing additional multilayer (ML) periods of a thin-film interference coating, distortions in the reflected wave front induced by surface figure errors can be corrected. At $\lambda=13.4 \mathrm{~nm}$ in the extreme-ultraviolet region the removal or deposition of a single period of the standard two-component molybdenum-silicon (Mo/Si) ML interference coating induces an effective phase change of magnitude $0.043 \pi$ with respect to an identical optical thickness in vacuum. The magnitude of this wave-front shift can be enhanced with multicomponent MLs optimized for phase change on reflection. We briefly discuss the contributions of the shift in the effective reflection surface of the ML on the phase change. We also predict the feasibility of novel phase-shifting masks for subwavelength imaging applications. (C) 2003 Optical Society of America
\end{abstract}

OCIS codes: $160.3900,230.4170,310.6860,340.7470$.

\section{Introduction}

The advent of extreme-ultraviolet (EUV) imaging with its applications in next-generation semiconductor lithography has necessitated the deployment of near-normal incidence, diffraction-limited optics requiring high wave-front integrity. ${ }^{1}$ This in turn requires that the error in the figure of the reflector be less than $1 / 16$ of the wavelength, giving a wave-front error in reflection of less than $\pi / 4 \mathrm{rad}$. In the 4-20-nm EUV spectral region the maximum acceptable figure errors range from 0.25 to $1.25 \mathrm{~nm}$ across the optic, which may be many centimeters in extent. At $\lambda \sim 13 \mathrm{~nm}$ a reflector figure error of $\sim 3 \mathrm{~nm}$ induces an error in the wave front of $\sim \pi \mathrm{rad}$, rendering the reflector useless for imaging purposes. Such errors may be systematic or random and may be due to a multitude of causes, e.g., substrate figure error, multilayer (ML) defects, ML-induced stress. It is therefore important that a postprocess phase-correction strategy be available to eliminate or reduce wave-

M. Singh, M. F. Bal, and J. J. M. Braat (J.J.J.Braat@TNW. TUDelft.NL) are with the Optics Research Group, Faculty of Applied Sciences, Deflt University of Technology, Lorentzweg 1, 2628 CJ Delft, The Netherlands. D. Joyeux is with the Laboratoire Charles Fabry, Institut d'Optique Théorique et Appliquée, BP 14791403 Orsay, France. U. Dinger is with Carl Zeiss SMT AG, Carl Zeiss Strasse, 73447 Oberkochen, Germany.

Received 12 July 2002; revised manuscript received 17 December 2002 .

0003-6935/03/101847-05\$15.00/0

(C) 2003 Optical Society of America front errors effectively. In this paper we describe a novel method for wave-front correction based on the correction ML, which is an improvement on the correction strategies proposed until now, e.g., the singlecorrection-layer method that we briefly outline. We also propose that the ML can be modified to act as a phase-shifting device for applications in EUV interferometry and subwavelength imaging.

\section{Numerical Methods}

In this study the thin-film calculations were performed with TFCalc (Software Spectra Inc.) and in the symbolic mathematical environment of Mathematica (Wolfram Research Inc.). The standard characteristic matrix method for thin-film calculations was employed. ${ }^{2}$ Implicit in this formalism is the assumption that an infinitely extending plane wave is incident on the ML. From the amplitude reflectivity $r$, obtained from the ML admittance, the phase change on reflection can be computed from $\arg (r)$ and the product of $r$ with its conjugate yields the intensity reflectivity $R$. We assume a normalincidence operation of the mirrors. The complex refractive indices of the various materials used in this study are taken from Henke et al., ${ }^{3}$ and their values are tabulated in Table 1.

\section{Single-Correction-Layer method}

Previously the property of materials in the EUV region having a complex refractive index of the form $\tilde{n}$ $=n-i k$, where $n \approx 1$, was proposed as a method whereby a film of a suitable material, thicker than 
Table 1. Optical Constants of the Materials ${ }^{a}$

\begin{tabular}{ccc}
\hline & \multicolumn{2}{c}{$\begin{array}{c}\text { Optical Constants } \\
(\lambda=13.4 \mathrm{~nm})\end{array}$} \\
\cline { 2 - 3 } Material & $n$ & $k$ \\
\hline Mo & 0.9227 & 0.0062 \\
Pd & 0.8780 & 0.0443 \\
Pt & 0.8928 & 0.0577 \\
Rh & 0.8775 & 0.0296 \\
Ru & 0.8898 & 0.0165 \\
Si & 0.9999 & 0.0018 \\
Y & 0.9742 & 0.0023 \\
\hline
\end{tabular}

${ }^{a}$ The complex refractive indices $(n-i k)$ are from Ref. 3 .

the figure error by a factor of $|1 /(1-n)|$, may be used to correct the wave front. ${ }^{4}$ The phase change $\Delta \phi$ through the film (ideally molybdenum for the 11$16-\mathrm{nm}$ region) is given by $\exp (-i \Delta \phi)=\exp (-4 \pi k d /$ $\lambda) \exp [-i 4 \pi(1-\mathrm{n}) \mathrm{d} / \lambda]$, where $d$ is the correctionlayer thickness and $\lambda$ is the vacuum wavelength. However, the decay term $\exp (-4 \pi k d / \lambda)$ induces a reflectivity loss, which is an unfortunate limitation of this approach. Another disadvantage of this method is that suitable correction layers in this spectral region, e.g., $\mathrm{Mo}, \mathrm{Ru}$, and $\mathrm{C}$, all exhibit anomalous dispersion, i.e., $n<1$. Such corrective layers may be used to correct for valleylike figure errors. Hill-like figure errors require materials with $n>1$. (This is shown schematically in the inset in Fig. 1.) Unfortunately, in the spectral region of interest, materials with $n>1$ exhibit prohibitively high absorption if employed as correction layers. In Fig. 1 we show the reflectivity and the phase change as a function of the correction-layer thickness of Mo (with respect to an identical optical path in vacuum). The results show, for example, that at $\lambda=13.4 \mathrm{~nm}$ a wave-front error of $\pi$ rad may be corrected by a 43 -nm Mo film $(n=0.9227)$, corresponding to a substrate error of $\lambda / 4 \mathrm{~nm}$ but at the expense of a loss of

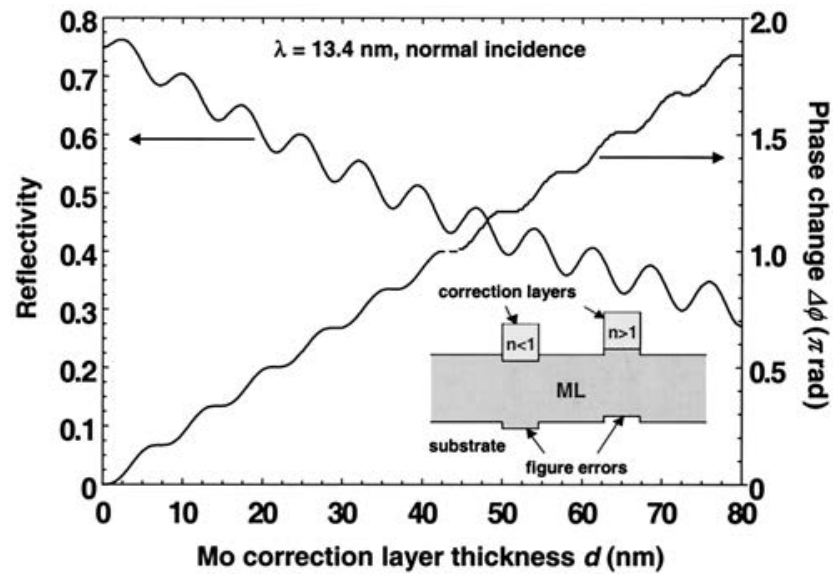

Fig. 1. Reflectivity and phase change as a function of the thickness of the Mo correction layer with reference to a vacuum layer of equal thickness. The inset shows schematically the correction methods for the two types of substrate figure error.

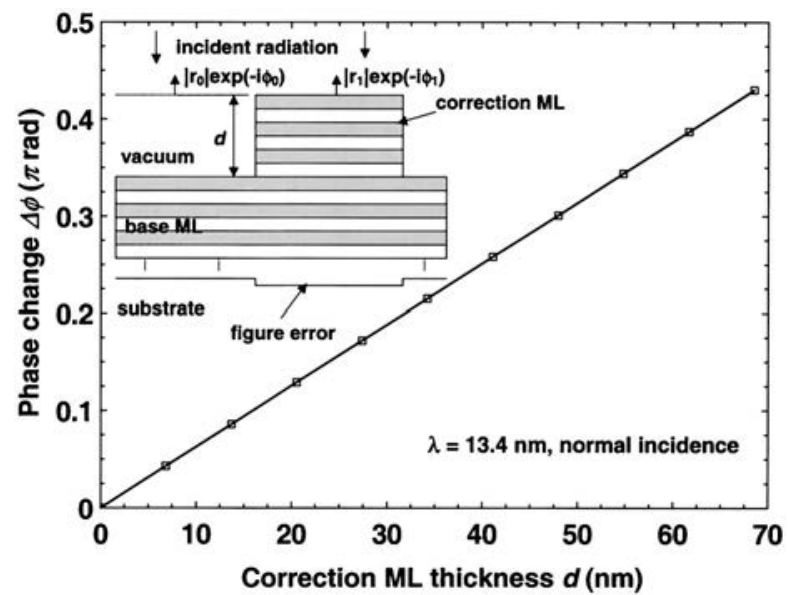

Fig. 2. Numerical simulation of the phase change with respect to a vacuum layer (inset) associated with $0-10 \mathrm{Mo} / \mathrm{Si}$ periods. The line through the data points is a least-squares fit. The inset is a schematic representation of the geometry and the associated phase shifts due to additional (or removed) ML periods. The correction ML of physical thickness $d$ corrects for the idealized figure error. The expressions $\left|r_{j}\right| \exp \left(-i \phi_{j}\right)$ are the amplitude reflectivities with their associated phase change on reflection.

0.25 in reflectivity. The typically high loss of reflectivity in this single-correction-layer method calls for an improved correction strategy.

\section{Correction-multilayer Method}

Recently Yamamoto ${ }^{5}$ proposed that removal by ionbeam milling of periods from a standard ML alters the phase change on reflection between the two surfaces with respect to an identical optical thickness in vacuum, called the vacuum layer. A phase change of $\sim 0.28 \pi$ for a total milled thickness of $\sim 65 \mathrm{~nm}(10$ periods) was demonstrated theoretically at $\lambda=12.8$ $\mathrm{nm}$.

We now introduce our improved approach to wavefront correction that we believe can be applied more universally than that proposed in Ref. 5. We compare the phase change on reflection of the base ML plus the vacuum layer of thickness $d$ with that of a base ML deposited with extra periods of total physical thickness $d$, as shown in the inset in Fig. 2. The net reflected wave-front change $\left(\Delta \phi=\phi_{0}-\phi_{1}\right)$ is given by $\arg \left(r_{0} / r_{1}\right)$, where $r_{0}$ is the amplitude reflectivity of the vacuum layer plus the standard base Mo/Si ML and $r_{1}$ is the amplitude reflectivity of the additional periods of the correction ML plus the base ML. The base ML is composed of 50 periods: 2.68 $\mathrm{nm}$ of Mo and $4.17 \mathrm{~nm}$ of $\mathrm{Si}$ (which is a ML designed for optimal reflectivity at $\lambda=13.4 \mathrm{~nm}$ with constant layer thicknesses) and a 2-nm capping layer of $\mathrm{SiO}_{2}$ native oxide. Optimized periods were numerically added one by one on the base ML and phase changes were recorded.

Figure 2 shows phase-change data for (as many as) 10 additional Mo/Si periods (each data point is an additional Mo/Si pair) on an optimized ML designed for a peak reflectivity at $\lambda=13.4 \mathrm{~nm}$ and normal- 
incidence operation as described in Refs. 6 and 7 . The data show that a phase correction of $0.43 \pi \mathrm{rad}$ can be achieved by adding $10 \mathrm{ML}$ periods of total physical thickness of $69 \mathrm{~nm}$ to the base ML. This value of phase change $(0.43 \pi)$ corresponds to a total path length of $2.88 \mathrm{~nm}$ in vacuum. Therefore 10 $\mathrm{Mo} / \mathrm{Si}$ periods can correct for a figure error of 1.44 $\mathrm{nm}$. Since the mean refractive index of the layers of the added $\mathrm{Mo} / \mathrm{Si}$ correction ML is less than unity, such a configuration corrects for valleylike figure errors, as for the case of the single Mo correction layer described in Section 3. In Fig. 2 the solid line is a least-squares fit to the data and shows the linear dependence of phase change with ML physical thickness. This correction method leads to an increase in the reflectivity of around $0.009 \%$ due to the addition of 10 optimized Mo/Si ML periods, as against an $\mathrm{R}=$ $0.12 \%-0.15 \%$ loss with a Mo single-layer correction strategy (Fig. 1). In addition for a nominal (0.02$0.06)$ loss in $R$ our computations show that a phase correction of $0.6 \pi-0.9 \pi$ may be achieved by varying the partition ratio and/or by using Ru instead of Mo and detuning one or more of the periods. In the latter case there may be a spectral shift in the reflectivity peak but at a tolerable level of $\leq 0.1 \mathrm{~nm}$. We have shown that optimum peak reflectivity may be achieved when ruthenium layers are incorporated within the ML.6,7 Note that multicomponent ML designs for the EUV region have more recently received further theoretical appraisal. ${ }^{8}$

A salient feature of this approach is that a phase change with an opposite sign can be obtained, depending on whether ML periods are added or subtracted from the stack. This can easily be deduced by considering the total ML above the idealized figure error shown in Fig. 2 as the base ML. If the corresponding surface on the substrate is set as a reference, hill-like errors may be corrected by removing ML periods relative to the top surface of the base ML; the phase change is now $\Delta \phi=\phi_{1}-\phi_{0}$. This means that this strategy can be applied to correct for both valleylike and hill-like figure errors.

In Fig. 3 we show some esoteric ML stacks employed as additive correction MLs similar to those elucidated in our earlier studies, ${ }^{6,7}$ some of which exhibit a significantly enhanced sensitivity to phase in relation to total physical thickness, i.e., the number of additional ML periods. For reference the data labeled $\mathrm{Mo} / \mathrm{Si}$ in Fig. 3 represent the standard 50period base Mo/Si ML as defined above with the phase change induced by additional periods of a corrective Mo/Si ML as in Fig. 2 and design 1 in Table 2. A significantly greater phase sensitivity may be achieved by using extra periods of a three-component $\mathrm{Ru}-\mathrm{Mo} / \mathrm{Si}$ stack with the $\mathrm{Ru}$ and Mo thickness kept constant at $2 \mathrm{~nm}$ for each, thus forcing a high phase change through the films on account of the low values of $n$ for Mo and Ru $(n \sim 0.9)$ but at the expense of 0.025 in reflectivity for 10 extra periods corresponding to $\Delta \phi \approx 0.75 \pi \mathrm{rad}$. Phase sensitivity may be further enhanced by using $\mathrm{Mo} / \mathrm{Y}$ as the additional

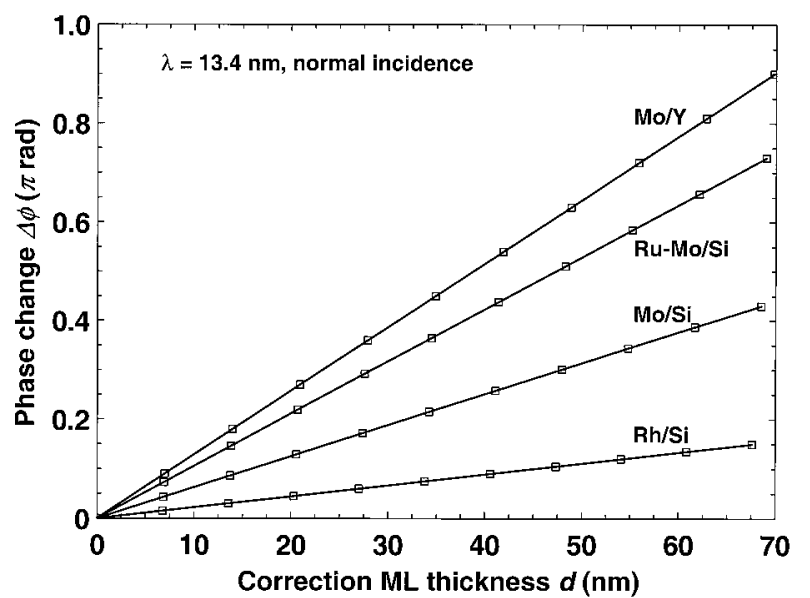

Fig. 3. Magnitude of the phase change associated with various ML stacks deposited on a base Mo/Si ML as a function of the physical thickness $d$. The data points correspond to $\Delta \phi$ at each complete additional ML period. The MLs correspond to designs 1 , 6,8 , and 11 in Table 2 .

periods at the expense of 0.05 in $R$ for 10 periods. These and other ML designs are tabulated in Table 2. The high phase sensitivity enables phase corrections to be achieved with a minimum change in physical thickness, a potentially useful property. In Table 2 the calculated phase sensitivities for the various ML structures are shown. The phase change per period $(\Delta \varepsilon)$ incurred by a wave traversing through one additional period is shown, again referring to a vacuum layer of the same optical thickness. The peak overall $\mathrm{R}$ is shown for 5 and 10 additional periods of each given structure. Note in these data that at the expense of reflectivity the additional periods may be optimized for optimal phase change with respect to the corresponding vacuum layer. This can be seen by comparing designs 1 and 2 where the phase sensitivity per period $(\Delta \varepsilon)$ increases from $0.043 \pi$ to $0.054 \pi$ as the Mo thickness is set to $4.0 \mathrm{~nm}$ in design 2 in Table 2.

$\mathrm{Ru} / \mathrm{Si}$ designs 3 and 4 exhibit a large change in $\Delta \varepsilon$ because the Ru thickness is varied from 1.98 to 3.50 $\mathrm{nm}$. This is due to the lower $n$ of Ru compared with Mo (Table 1). However, the high extinction coefficient $k$ of $\mathrm{Ru}$ means that the peak reflectivity drops significantly. The three-component $\mathrm{Ru}-\mathrm{Mo} / \mathrm{Si} \mathrm{MLs}$ maintain high peak reflectivity even as the $\mathrm{Ru}$ and Mo thickness is increased, with designs 6 and 7 in Table 2 exhibiting particularly high values of $\Delta \varepsilon$. The yttrium-based MLs exhibit the largest phase sensitivity owing to the significant contribution of $\mathrm{Y}$ to the phase change with respect to the vacuum layer, as is evident by its refractive index being lower than that of Si (Table 1). Designs 1, 6, 8, and 11 of Table 2 are shown in Fig. 3. Designs 13 and 14 are those in which the Si or Y thickness is detuned so that the position of the peak reflectivity shifts from 13.4 to $13.5 \mathrm{~nm}$. However, there is a marked increase in the phase-change sensitivity in these designs. The re- 
Table 2. Phase Sensitivity and Effective Peak Reflectivity of Various Correction ML Designs Deposited on an Optimized 50-Period Mo/Si Base ML Tuned for $\lambda=13.4-\mathrm{nm}$ Operation with a Peak $R$ of 0.748

\begin{tabular}{|c|c|c|c|c|c|c|c|}
\hline \multirow[b]{2}{*}{ Design } & \multirow{2}{*}{$\begin{array}{l}\text { ML Period of the } \\
\text { Form } x-y / z\end{array}$} & \multicolumn{3}{|c|}{ Layer Thickness (nm) } & \multicolumn{2}{|c|}{ Peak Reflectivity } & \multirow[b]{2}{*}{$\Delta \varepsilon^{a}(\pi \mathrm{rad})$} \\
\hline & & $x$ & $y$ & $z$ & 5 Periods & 10 Periods & \\
\hline 1 & $\mathrm{Mo} / \mathrm{Si}$ & - & 2.68 & 4.17 & 0.749 & 0.749 & 0.043 \\
\hline 2 & $\mathrm{Mo} / \mathrm{Si}$ & - & 4.00 & 2.90 & 0.725 & 0.716 & 0.054 \\
\hline 3 & $\mathrm{Ru} / \mathrm{Si}$ & - & 1.98 & 4.82 & 0.727 & 0.716 & 0.028 \\
\hline 4 & $\mathrm{Ru} / \mathrm{Si}$ & - & 3.50 & 3.40 & 0.678 & 0.658 & 0.080 \\
\hline 5 & $\mathrm{Ru}-\mathrm{Mo} / \mathrm{Si}$ & 1.00 & 1.68 & 4.18 & 0.752 & 0.754 & 0.045 \\
\hline 6 & $\mathrm{Ru}-\mathrm{Mo} / \mathrm{Si}$ & 1.80 & 1.80 & 3.30 & 0.737 & 0.734 & 0.073 \\
\hline 7 & $\mathrm{Ru}-\mathrm{Mo} / \mathrm{Si}$ & 2.00 & 2.00 & 2.93 & 0.723 & 0.715 & 0.090 \\
\hline 8 & $\mathrm{Rh} / \mathrm{Si}$ & - & 1.51 & 5.25 & 0.704 & 0.679 & 0.015 \\
\hline 9 & $\mathrm{Pd} / \mathrm{Si}$ & - & 1.15 & 5.59 & 0.688 & 0.648 & 0.008 \\
\hline 10 & $\mathrm{Pt} / \mathrm{Si}$ & - & 0.87 & 5.85 & 0.678 & 0.625 & 0.003 \\
\hline 11 & $\mathrm{Mo} / \mathrm{Y}$ & - & 2.61 & 4.37 & 0.695 & 0.664 & 0.090 \\
\hline 12 & $\mathrm{Ru}-\mathrm{Mo} / \mathrm{Y}$ & 2.00 & 2.00 & 3.06 & 0.685 & 0.639 & 0.109 \\
\hline 13 & Ru-Mo/Y & 2.00 & 2.00 & 3.15 & 0.670 & 0.626 & 0.121 \\
\hline 14 & Ru-Mo/Si & 2.00 & 2.00 & 3.10 & 0.658 & 0.608 & 0.111 \\
\hline
\end{tabular}

${ }^{a}$ Phase change per ML period with reference to the vacuum layer.

flectivity values quoted for designs 13 and 14 are for a design wavelength of $13.4 \mathrm{~nm}$.

We consider again the phase change induced by 10 additional Mo/Si periods on the standard 50-period Mo/Si ML stack. The numerical data for this configuration are presented as design 1 in Table 2 and are also presented in Fig. 2. The data show that 10 deposited periods change the phase of the emergent wave front by $0.43 \pi$ rad with respect to the base ML and the vacuum layer. The 10 additional periods contain a total of $26.8 \mathrm{~nm}$ of Mo and $41.7 \mathrm{~nm}$ of Si. Considering these total thicknesses separately and taking the values of $n$ for Mo and Si from Table 1, one predicts a total phase change $[\Delta \phi=4 \pi(1-n) d / \lambda]$ of $0.62 \pi \mathrm{rad}$. Since $\Delta \phi(\mathrm{Si}) \approx 0$ this value is the mean phase-change value for the single Mo correction layer (Fig. 1).

The discrepancy of $0.19 \pi \mathrm{rad}$ in the wave-front phase change may be explained in part by taking into account the depth of the effective reflection surface (ERS) within the ML. The concept of ERS requires that the ML be replaced with a single surface with a certain admittance and reflection phase generated by the additive multiple reflections and phase changes within the ML. In general, to do this, we have to consider the group velocity of a finite spectral wave packet, and then the position of the effective reflection surface is a function of the group delay, which is proportional to the derivative of the phase. ${ }^{9}$ The effective depth of reflection in a multilayer may also be approached as the average depth of reflection of all reflected fields. ${ }^{10}$ From the results in Ref. 10 we present data on the evolution of the depth of the ERS as a function of the number of periods of a Mo/Si ML (Fig. 4). The depth approaches saturation at around 50 periods, corresponding well to the saturation of the peak reflectivity. The inset in Fig. 4 shows the saturation region between 40 and 60 periods. These data show that when an additive ML correction strategy is employed such that 10 periods are added to a 50-period ML, a small but significant change in the ERS depth of $\sim 0.34 \mathrm{~nm}$ takes place corresponding to a phase change of $0.1 \pi \mathrm{rad}$, which accounts for around half of the discrepancy of $0.19 \pi \mathrm{rad}$ mentioned above. Considering that there is modulation in $\Delta \phi$ of $\sim 0.1 \pi$ (Fig. 1), which together may account for the entire discrepancy, it is reasonable to conclude that the change in the ERS depth must be considered a major factor in a ML correction strategy and by inference on the imaging properties of EUV ML reflective optics. The large slope of the curve in Fig. 4 at low numbers of ML periods indicates a greater shift in the effective reflection surface in the ML. This effect may be utilized in correction strategies; e.g., to minimize the effect of the shift of the ERS, the ML must be operated well into its saturation regime. On the other hand, the ML may be operated below the reflectivity saturation regime to achieve a larger phase correction, taking advantage of the larger per

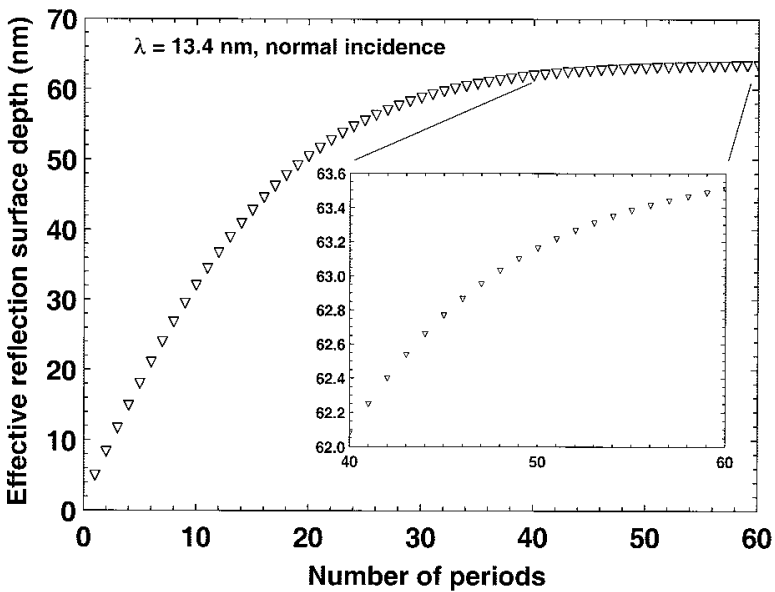

Fig. 4. Evolution of the depth of the effective reflection surface of a Mo/Si ML as a function of the number of periods. The inset is the expanded scale between 40 and 60 periods. 


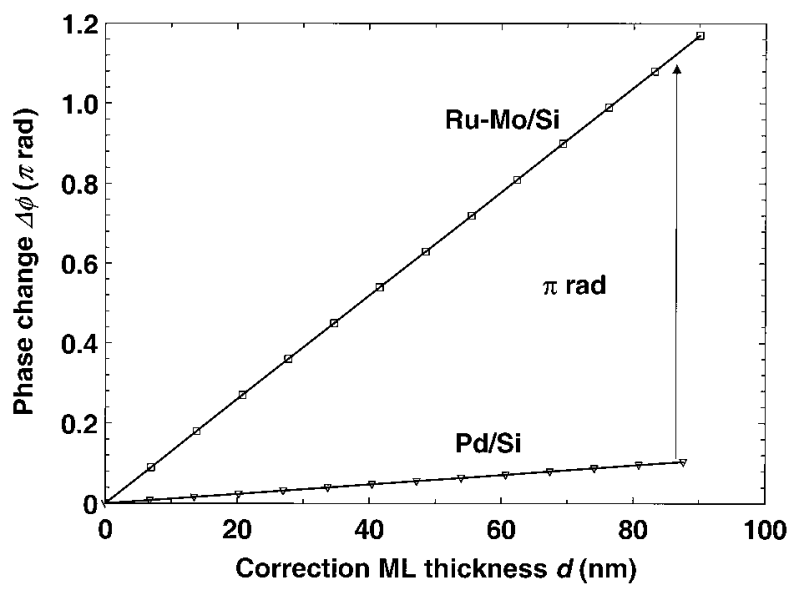

Fig. 5. Difference in the phase shifts due to the two MLs deposited on the base ML. A $\pi$-rad phase difference in the wave front is achieved after 12 periods.

period shift of the effective reflection surface in this regime. The inset in Fig. 4 shows that there is a greater change in the ERS depth between 40 and 50 periods than from 50 to 60 periods. More detailed data on these and other, particularly imaging, aspects of EUV MLs are in Ref. 10.

\section{Extreme-Ultraviolet Phase-Shifting Masks}

Another notable aspect of the results of this study is the small phase sensitivity of the $\mathrm{Rh} / \mathrm{Si}, \mathrm{Pd} / \mathrm{Si}$, and $\mathrm{Pt} / \mathrm{Si}$ designs (Table 2). This means that even a large physical thickness of the ML may be deposited, yielding a negligible wave-front phase change. This result has led us to predict the possibility of fabricating phase-shifting masks (PSMs) for EUV lithography. PSMs are presently employed in lithographic systems, enabling sub wavelength features to be delineated. This entails shifting the optical wave front by as much as $\pi$ rad. We have shown that with a number of designs a $\pi$-rad phase change is readily achievable with respect to the vacuum layer or with respect to a null-phase-change ML (e.g., designs 8, 9, and 10 in Table 2) albeit at the expense of reflectivity. A PSM design with a $\pi$-rad phase change with respect to a null ML means that the surface of the mask is level to within $\sim 3 \mathrm{~nm}$. In Fig. 5 a $\pi$ phase shift is shown between the 2 MLs after approximately 12 periods. The MLs shown are designs 7 and 9 in Table 2. We believe this to be the first proposal for PSMs in the EUV region by the removal or additional growth of a ML. We achieve this by optimizing the MLs for phase change and benefiting from additional materials and multicomponent MLs with suitable properties.

\section{Summary}

We have shown that the wave-front correction of the EUV reflectors may be performed by the removal or addition of ML periods. By using novel multicomponent MLs and by varying the partition ratio of the components of the ML, a flexible method of phase correction may be realized. The phase sensitivity of any such system may be optimised as required for any particular application. These new aspects lend themselves to zero or low-reflectivity-loss correction strategies crucial for EUV optics. We have also shown that the effective reflection surface of a ML has profound effect on the relative reflection phase. In addition we have proposed ML modification as a method that lends itself to the fabrication of phaseshifting masks for subwavelength imaging in the EUV spectral region.

This research was supported by the Directorate General for Industry of the European Commission through the European Union's Program on Information Technology (ESPRIT), project code EP-28146CALCORR.

\section{References}

1. C. W. Gwyn, R. Stulen, D. Sweeney, and D. Attwood, "Extreme ultraviolet lithography," J. Vac. Sci. Technol. B 16, 3142-3149 (1998).

2. H. A. Macleod, Thin-Film Optical Filters, 2nd ed. (Adam Hilger, Bristol, UK, 1986), pp. 11-43.

3. B. L. Henke, E. M. Gullikson, and J. C. Davis, "X-ray interactions: photoabsorption, scattering, transmission, and reflection at $E=50-30,000 \mathrm{eV}, Z=1-92$," At. Data Nucl. Data Tables 54, 181-342 (1993), http://www-cxro.lbl.gov/optical_constants.

4. J. J. M. Braat, "Phase correcting layers in EUV imaging systems for microlithography," in Extreme Ultraviolet Lithography, G. Kubiak and D. Kania, eds., Vol. 4 of OSA Trends in Optics and Photonics Series (Optical Society of America, Washington, D.C., 1996), pp. 152-155.

5. M. Yamamoto, "Sub-nanometer figure error correction of an extreme ultraviolet multilayer mirror by its surface milling," Nucl. Instrum. Methods Phys. Res. A 467-468, 1282-1285 (2001).

6. M. Singh and J. J. M. Braat, "Design of multilayer extremeultraviolet mirrors for enhanced reflectivity," Appl. Opt. 39, 2189-2197 (2000).

7. M. Singh and J. J. M. Braat, "Improved theoretical reflectivities of extreme-ultraviolet mirrors," in Emerging Lithographic Technologies IV, E. A. Dobisz, ed., Proc. SPIE 3997, 412-419 (2000).

8. J. I. Larruquert, "Reflectance enhancement in the extreme ultraviolet and soft $\mathrm{x}$ rays by means of multilayers with more that two materials," J. Opt. Soc. Am. A 19, 391-397 (2002).

9. C. K. Madsen and J. H. Zhao, Optical Filter Design and Analysis (Wiley Interscience, New York, 1999), p. 285.

10. M. F. Bal, F. Bociort, and J. J. M. Braat, "The influence of multilayers on the optical performance of extreme ultraviolet projection systems", in International Optical Design Conference 2002, P. K Manhart and J. M. Sasian, eds., Proc. SPIE 4832, 149-157 (2002). 